\title{
A Typical Case of Bouveret's Syndrome, or Not?
}

\author{
Gusta van Zwieten ${ }^{1}$, Eric B. de Goede ${ }^{2}$, Ronald T. van der Laan ${ }^{3}$, Jeroen Heemskerk
}

1) Department of Surgery; 2) Department of Hepatogastroenterology; 3) Department of Radiology, Laurentius Hospital Roermond, The Netherlands
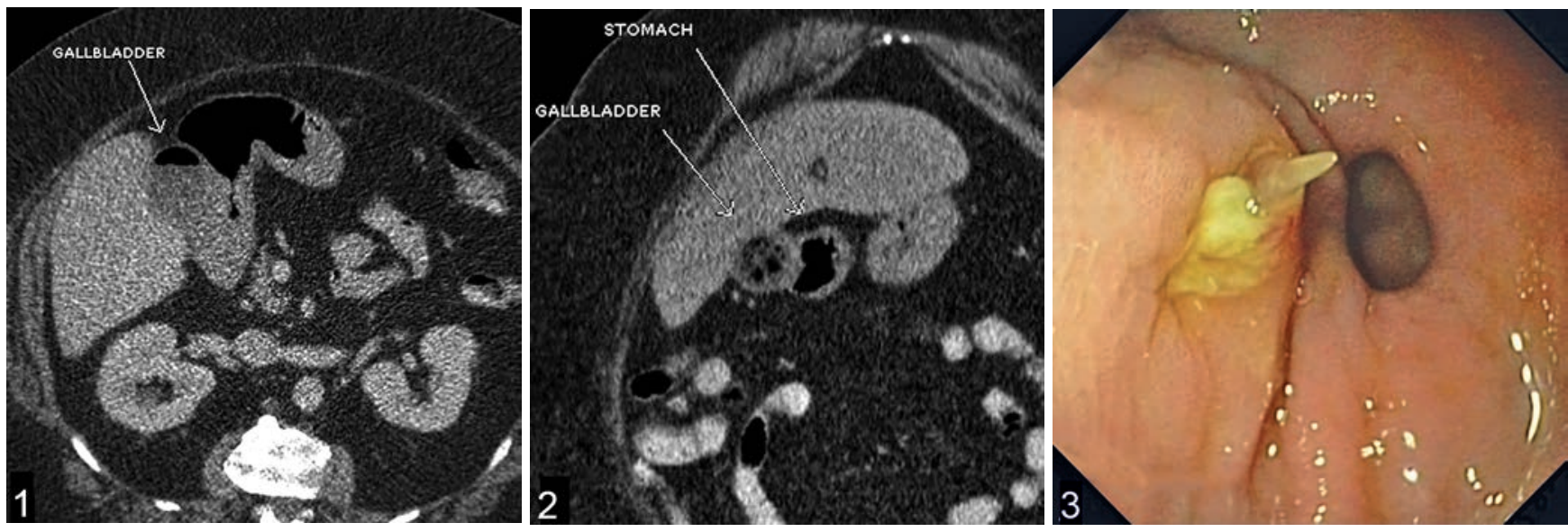

A 68-year-old obese woman (BMI $\left.57 \mathrm{~kg} / \mathrm{m}^{2}\right)$ presented with continuous abdominal pain and extensive vomiting since five days. Physical examination revealed sparse peristaltic sounds, abdominal tenderness but no muscular defense. Laboratory examination showed increased C-reactive protein $(79 \mathrm{mg} / \mathrm{L})$ and leucocytosis $\left(17.5 \times 10^{9} / \mathrm{L}\right)$. Liver function tests were normal.

CT scan demonstrated an air-fluid level inside the gallbladder (Fig. 1, axial MDCT slice in late portal-venous phase) and a thickened gallbladder wall, which was not sharply delineated from the stomach wall (Fig. 2, coronal MDCT slice). These findings suggested fistulisation from the gallbladder to the duodenum or stomach, presumably by a gallstone. Bowel obstruction could not be objectivated. Under the diagnosis of "Bouveret's syndrome", a gastroduodenoscopy was performed in order to confirm the diagnosis and clear the duodenum from possible gallstones. Surprisingly, gastroduodenoscopy did not show gallstones, but revealed a sharp piece of plastic, stuck in the antrum of the stomach. The prepyloric gastric wall showed edema and puss excretion from the perforation site (Fig. 3). The foreign body could be removed successfully. After one month of follow-up, the patient was completely recovered. She mentioned that she had probably ingested a plastic wrapping of a deep frozen pizza.

Ingestion of a foreign body does not cause any symptoms in $80-90 \%$ of the cases. When symptoms arise they are usually secondary to obstruction or inflammation. Gastrointestinal perforation by ingested foreign bodies occurs in less than $1 \%$ of patients. Diagnosis is difficult due to multiple reasons. Patients are often unaware of ingestion of a foreign body and the clinical presentation and routine laboratory studies are nonspecific. Unless the foreign body is radio-opaque it will not be identified on radiography [1]. In our case, the CT-scan demonstrated images seen in Bouveret's syndrome. In this syndrome, gallstones migrate through a cholecystoduodenal fistula caused by calculous cholecystitis, resulting in nausea and vomiting due to proximal intestinal obstruction [2]. Other known causes of cholecystoenteric fistulas are duodenal ulcers, gallbladder neoplasm, inflammatory bowel disease and diverticular disease [3]. Perforation of the gallbladder by an ingested foreign body, clinically and radiologically mimicking Bouveret's syndrome, seems to be extremely rare.

Corresponding author: Jeroen Heemskerk; jeroen.heemskerk@lzr.nl

Conflicts of interest: None to declare.

\section{REFERENCES}

1. Santos SA, Alberto SC, Cruz E, et al. Hepatic abscess induced by foreign body: case report and literature review. World J Gastroenterol 2007;13:1466-1470.

2. Cappell MS, Davis M. Characterization of Bouveret's syndrome: a comprehensive review of 128 cases. Am J Gastroenterol 2006;101:21392146.

3. Dan D, Collure DW, Hoover EL. Bouveret's syndrome: revisiting gallstone obstruction of the duodenum. J Natl Med Assoc 2003;95:969973. 